\title{
B-scan analysis of subsurface radar sounding of lunar highland region
}

\author{
Takao Kobayashi $^{1}$, Hiroshi Oya ${ }^{2}$, and Takayuki Ono ${ }^{1}$ \\ ${ }^{1}$ Tohoku University, 980-8578, Japan \\ ${ }^{2}$ Fukui University of Technology, 910-4272, Japan
}

(Received December 28, 2001; Revised September 13, 2002; Accepted September 29, 2002)

\begin{abstract}
Subsurface sounding of lunar highland region by Lunar Radar Sounder of SELENE project has been investigated based on a computer simulation technique which enables us to analyze sounder echo signals from a modeled lunar surface. Using a numerically generated surface feature of a highland region which is characterized by impact craters, whole sequence of LRS observations, and data analyses have been simulated. The study was carried out on those simulated LRS observation data in order to establish a data analysis methodology of subsurface sounding in highland region. The established data analysis methodology consists of a set of data analyses, and gives a guide line to determine the detectability of subsurface signal. The points of the methodology are (1) the subsurface echo signal is recognized as a straight linear pattern in B-scan display, and (2) data stack technique is introduced to reduce surface off-nadir echoes, however, (3) the data stack should be done within the range of optimal data stack, and, (4) the limit of optimal data stack is determined from the behavior of the surface nadir echo.
\end{abstract}

\section{Introduction}

This paper is the companion paper to Kobayashi et al. (2002) concerning to data analysis methodology of the Lunar Radar Sounder (LRS) mission of Japanese lunar exploration project, SELENE. The primary objective of LRS is to investigate lunar geologic subsurface structure.

The most concerning problem lies in the feasibility of LRS observation in highland regions which occupy more than $80 \%$ of the lunar surface because of their ragged surface feature of heavily cratered terrain. Such a rough surface is thought to reflect strong backscattering echoes, or surface clutters, which would prevent weak subsurface echoes from being detected. In fact, no successful result concerning to highland region has been published from Apollo Lunar Sounder Experiment (ALSE) that has been the only attempt of the similar kind to the LRS ever made (Phillips et al., 1973).

ALSE data analysis suffered two disadvantages: one was that all the observation data was recorded in optical films (analog storage device), which required to use complex optical devises in data handling, another was that phase information of signal was not utilized in their analysis except in an experimental attempt where a small portion (2 MB) of observation data was digitized from optically recorded data (Phillips et al., 1973). Those disadvantages eventually limited ALSE data analysis to single pulse intensity analysis in which weak subsurface echoes were hardly distinguished if they were overlapped by stronger surface clutters.

Three decades have passed since ALSE, and the dominant technology in signal processing has shifted from analog domain to digital technology domain. In the LRS mission, all

Copy right (C) The Society of Geomagnetism and Earth, Planetary and Space Sciences (SGEPSS); The Seismological Society of Japan; The Volcanological Society of Japan; The Geodetic Society of Japan; The Japanese Society for Planetary Sciences. the observation data is to be handled and analysed in fully digital manner, which enables much more flexible and finer data handling in both recording and analysing than analog technology would.

We have studied a data analysis technique for subsurface sounding observation of LRS in highland regions so as to maximize the detectability of subsurface signal. Since no real data of LRS observation has been available yet, we carry out computer simulation of LRS observation of a highland region with a newly developed simulation code, the Kirchhoff-approximation Sounding Simulation (KiSS) code (Kobayashi et al., 2002). The surface feature of the highland region is numerically generated in the simulation.

\section{Simulation Model and Simulation Condition 2.1 Surface model}

In the present study, the lunar surface feature of a highland region is modeled as a cratered terrain which is characterized by impact craters. Those impact craters are numerically generated by a subprogram of the KiSS code following two statistical properties of lunar impact craters: one is that of crater population and another is of crater morphology.

Baldwin (1964) showed that the cumulative count of lunar crater follows a power law with respect to the crater diameter. He deduced the power law of total cumulative production of craters described as

$$
\log N_{\text {cum }} \propto-2.12025 \log D
$$

where $N_{\text {cum }}$ is the cumulative crater number whose diameter is greater than $D$. We adopt this empirical formula with a little modification as

$$
\log N_{\text {cum }} \propto-2 \log D
$$

to characterize a statistical property of numerically generated impact craters in the present simulation. 
As for crater morphology, Pike (1974) found a strong correlation between crater diameter and crater depth after making measurements of crater morphology in a wide range of dimension based on the data from maps and profiles which had been compiled photogrammetrically from Apollo 15, 16, and 17 photographs: the depth of craters increases linearly as the diameter increases, keeping the ratio of depth/diameter of 0.2 up to $15 \mathrm{~km}$ in diameter over which the ratio tends to decline.

The shape of a crater had been found to depend on its diameter, and classifications of lunar crater morphology were published by authors (see reference of McGill, 1974). In our simulation, a simple model of impact crater morphology is employed: a crater whose diameter is smaller than $10 \mathrm{~km}$ has the bowl shape while a larger crater has the flat bottom shape, and any crater has axisymmetric shape. They are defined by sets of formulae which define the elevation of crater slope as functions of distance from the center of the crater, $r$.

The set of formulae for a crater whose diameter is smaller than $10 \mathrm{~km}$ is

$$
z(r)=\left\{\begin{array}{c}
{\left[(\alpha+\beta \ln R)+\gamma\left\{\exp \left(\frac{r-R}{R}\right)-1\right\}\right]} \\
\times \frac{R}{1-e^{-1}} \quad(0 \leq r \leq R) \\
(\alpha+\beta \ln R) \exp \left[-\frac{(r-R)^{2}}{0.64 R^{2}}\right] R \\
(R<r \leq a R)
\end{array}\right.
$$

where $z(r)$ is the elevation of the crater slope at distance $r$ from the centre of the crater whose radius is $R$, and $\alpha, \beta$, and $\gamma$ are constants that characterize the shape:

$$
\alpha=\frac{\ln 2}{40 \ln 10}, \quad \beta=\frac{1}{40 \ln 10}, \quad \gamma=0.25 .
$$

$a$ is also a constant which limits the extension of the crater skirt and is chosen to be 2.2 .

For those craters whose diameter is greater than $10 \mathrm{~km}$, the formulae are as follow:

$$
z(r)= \begin{cases}u \exp \left[-\frac{(r-R)^{2}}{v^{2}}\right]-w & (0 \leq r \leq R) \\ p \exp \left[-\frac{(r-R)^{2}}{q^{2}}\right] & (R<r \leq a R)\end{cases}
$$

where $u, v, w, p$, and $q$ are functions of $R$ being defined as

$$
u=1.690 R^{0.23}, \quad v=1.352 R^{0.23}, \quad w=1.268 R^{0.23},
$$

and

$$
p=0.422 R^{0.23}, \quad q=3.380 R^{0.23} .
$$

The generation process of craters is assumed to be impact cratering due to meteorite bombardments. The position of an impact point on the simulation plane is chosen at random by Monte Carlo method. The dimension of a crater is chosen at random as well, but keeping the statistical property of cumulative crater number. This production process is described as

$$
R_{n}=R_{\min } / \sqrt{a_{n}}
$$

where $R_{n}$ is the rim radius of the $n$th crater produced, $R_{\min }$ is the radius of the minimum crater produced and $a_{n}$ is the value of the $n$th uniform random number in the range

$$
0<a_{n} \leq 1
$$

The uniform random number $a_{n}$ is produced by means of linear congruential method. The repetition period of the random number is $\left(2^{31}-2\right)$ since the data length in the computer is 32 bit. This period allows us to produce independent craters more than $2 \times 10^{9}$.

We also take into account the effect of superposition and deterioration of craters, i.e. a younger crater may be produced on older craters and deteriorate a part of older ones or whole body of them if the dimension of the younger crater is large enough.

\subsection{Subsurface model}

Subsurface space consists of two layers as shown in Fig. 1. The subsurface boundary interface is assumed to be a smooth plane which is parallel to the mean surface or the upper boundary interface of the upper layer. The subsurface boundary interface is set at the depth of 1,250 m. Each layer is filled with uniform dielectric material.

\subsection{Simulation condition}

Simulation conditions are common to those of the simulation of LRS observation of lunar mare region as presented in Table 1 of Kobayashi et al. (2002), except those conditions which are specifically set for the case of highland region: the dielectric constant of the subsurface material of the upper layer is chosen to be $4.0+i 0.02,4.0+i 0.03$, and $4.0+i 0.05$ to represent slightly lossy media, moderately lossy media, and seriously lossy media, respectively. The dielectric constant, $\varepsilon_{2}$, of the lower layer material is assumed to be $8.0+i 0.05$.

In the simulation, the total number of 500 pulses of consecutive LRS observations were carried out along an orbit,

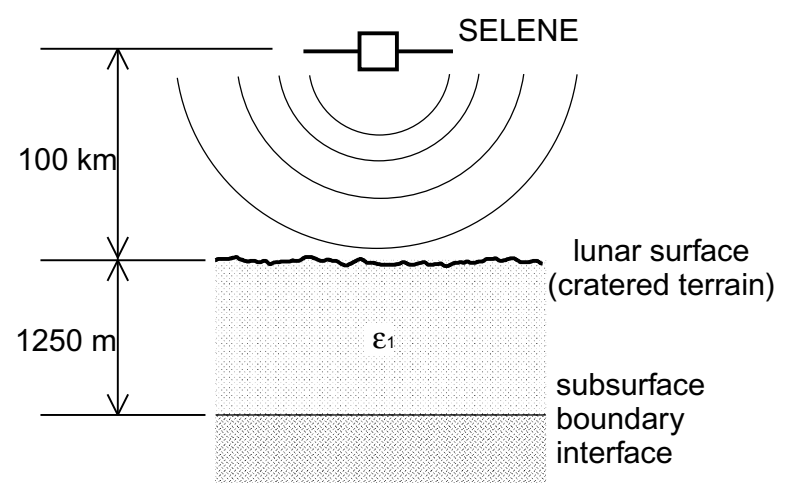

$\varepsilon 2$

Fig. 1. A schematic sketch of the lunar model. The surface is covered with impact craters. Subsurface space is divided into two layers by a horizontal subsurface boundary. The subsurface boundary interface is a smooth plane which is located at the depth of $1,250 \mathrm{~m}$. The upper layer is filled with uniform dielectric material whose dielectric constant, $\varepsilon_{1}$ is $4.0+i 0.02$ for slightly lossy subsurface material, $4.0+i 0.03$ for moderately lossy subsurface material, and $4.0+i 0.05$ for seriously lossy subsurface material. The lower layer is also filled with uniform dielectric material whose dielectric constant, $\varepsilon_{2}$, is $8.0+i 0.05$. 


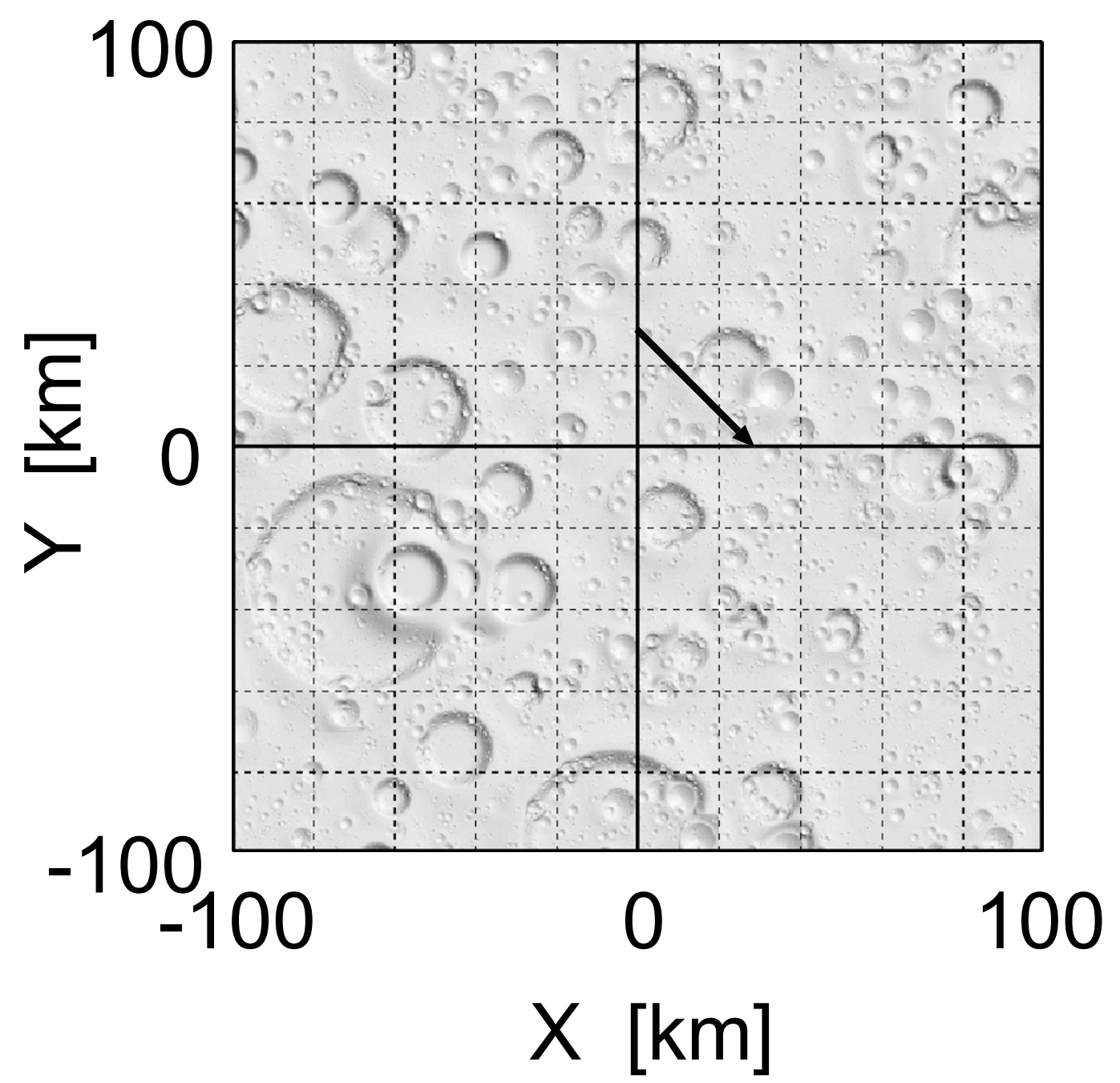

Fig. 2. An optical image of the model lunar surface. The surface is covered with impact craters with the population density of $100 \mathrm{~km}^{-2}$. The minimum radius of crater is $10 \mathrm{~m}$. An arrow in the figure indicates the ground track of the SELENE orbit. The length of the arrow corresponds to the move during the LRS observation period, $40 \mathrm{~km}$.

at the altitude of $100 \mathrm{~km}$ which is parallel to the mean surface. The time elapsed in the observation period is $25 \mathrm{sec}$. The speed of SELENE spacecraft is $1.6 \mathrm{~km} / \mathrm{sec}$, hence, the spacecraft moves $40 \mathrm{~km}$ during the period of 500 pulse observation. The orbit direction of the SELENE spacecraft in the simulation space is aligned to a diagonal direction of the square simulation surface. The ground track of the orbit is indicated in Fig. 2.

\section{Simulation}

\subsection{LRS observation of highland region}

Figure 3 shows an A-scope display of an LRS observation of highland region. The dielectric constant of the upper subsurface layer, $\varepsilon_{1}$, is $4.0+i 0.02$.

The range of the A-scope in Fig. 3 is offset for $100 \mathrm{~km}$ so that the zero range coincides with the range to the nadir point of the mean surface (the altitude of the SELENE orbit is $100 \mathrm{~km}$ ), therefore, the range is read as the apparent depth of a subsurface target as well as the offset range of a surface target.

The propagation speed, $v$, of an electromagnetic wave in a dielectric medium depends on its dielectric constant, $\varepsilon$, as

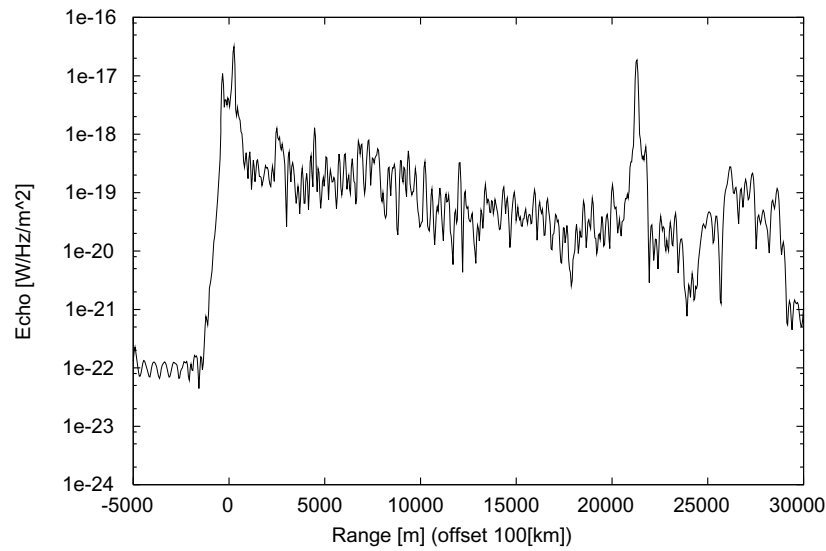

Fig. 3. An A-scope display of the case of slightly lossy subsurface material. A single pulse A-scope of LRS observation of Fig. 2. The observation point is $100 \mathrm{~km}$ above the point of $(X, Y)=(14.142 \mathrm{~km}, 14.142 \mathrm{~km})$ in Fig. 2. The range of the A-scope is offset $100 \mathrm{~km}$ so that the zero range coincides with the nadir point on the mean surface. Note that it impossible to distinguish the subsurface echo in this A-scope display. 


$$
v=\frac{c}{\sqrt{\Re \varepsilon}},
$$

where $c$ is the speed of light. Since the real part of $\varepsilon_{1}$ is 4.0 in the present simulation, the subsurface echo which is reflected at the boundary interface at the depth of $1,250 \mathrm{~m}$ should be found at the apparent depth of $2,500 \mathrm{~m}$, because the target range is calculated on the assumption that LRS pulse propagates at the speed of light, $c$, in both vacuum and in lunar subsurface material. Seeing the A-scope, it is obvious that surface clutter echoes are so intense at any range that subsurface echo is not discernible. Those surface clutter echoes come from random slopes which are produced by randomly scattered numerous craters, and are the same phenomena as we have seen in sounding observation of Gaussian random rough surface (Kobayashi et al., 2002). This feature gives the major problem on the practicability of lunar subsurface sounding in highland regions.

In addition, a cratered surface gives rise to unique phenomena when normal incidence of LRS pulse takes place. An example is the significantly intense echo that is observed at the range of $21,000 \mathrm{~m}$ in the A-scope of Fig. 3. The target location of the echo has been determined to be $(X, Y, Z)=(76 \mathrm{~km},-6 \mathrm{~km},-2 \mathrm{~km})$ in the coordinate of Fig. 2, where overlapping craters show a rather complex surface feature. The negative value of $Z$ coordinate refers to that the target location is inside a large crater where the surface altitude is lower than the mean surface. This kind of intense surface echoes will be also the obstacle in the detection of subsurface echoes.

However, a solution to the problem could be found by considering the nature of surface clutters in an A-scope display. The range of a surface clutter echo varies as the spacecraft makes orbital motion while the range of the subsurface echo would change little if the slope of the subsurface boundary interface is very small as is for the case of the present simulation.

Therefore, if a time series of A-scope data is made, the subsurface echo would be identified as an echo whose apparent range does not change while surface clutters, which lay over the subsurface echo, would change their ranges as functions of time.

The data set of time series of A-scope data is called B-scan data. We consider B-scan data of LRS observation, rather than the A-scope data, to distinguish subsurface echoes from surface clutter echoes.

Another problem to be considered is propagation attenuation in subsurface media. In the following sections, we study the effect of attenuation on LRS sounder signals during the propagating in the lunar subsurface. We examine cases of different degree of propagation attenuation in the subsurface media (upper layer), i.e. slightly lossy $\left(\varepsilon_{1}=4.0+i 0.02\right)$ and moderately lossy $\left(\varepsilon_{1}=4.0+i 0.03\right)$ conditions.

\subsection{LRS observation through slightly lossy subsurface material}

The dielectric constant of the subsurface material in the upper layer (Fig. 1) is assumed to be

$$
\varepsilon_{1}=4.0+i 0.02
$$

whose loss $\tan \delta$ is 0.005 . The dielectric constant of subsurface material in the lower layer is assumed to be

$$
\varepsilon_{2}=8.0+i 0.05
$$

Figure 4 shows the B-scan display of LRS observation results. In the B-scan display, we can identify the reflection echoes as classified in four categories:

(a) Surface nadir echo

The surface nadir echo appears around zero range with large intensity. A distinct difference of heavily cratered terrain from a mare surface is that surface nadir echo appear to move around the zero range. Sometimes it even splits to appear in two different ranges near zero range at a time, due to the surface terrain variation in the SELENE footprint.

(b) Intense surface off-nadir echo (strong surface clutter) Intense surface off-nadir echoes come from crater slopes where normal incidence of LRS pulse occurs. The echoes draw obvious patterns of hyperbolic curves in the B-scan display because their position relative to the SELENE spacecraft varies as the spacecraft moves.

(c) Weak surface off-nadir echo (diffuse surface clutter) The weak surface off-nadir echoes appear in all ranges. They are caused by surface roughness which was made by numerous small craters of random distribution. The nature of these echoes are similar to those from a random rough surface previously discussed by Kobayashi et al. (2002).

(d) Subsurface nadir echo

The subsurface nadir echo appears at a constant apparent range, 2,500 m, because the both subsurface boundary interface and the SELENE orbit are parallel to the mean surface plane. The pattern of the straight line gives a strong support to identify subsurface echoes in the presence of intense surface off-nadir echoes which appear as hyperbolic curve patterns in B-scan display.

As Fig. 4 shows, B-scan display is an effective data format to distinguish subsurface echo from surface off-nadir echoes.

Analysing LRS observation data of mare region in simulation, we have confirmed that the data stacking technique of waveform data is an effective way of reducing surface offnadir echo intensity (Kobayashi et al., 2002). The technique may also be applied to observation of highland region. Figure 5 presents a B-scan display of which each A-scope data is made from a mean waveform data of 11 consecutive observations. Obviously, 11 data stack reduces intensity of surface off-nadir echoes and, as a result, subsurface echo at the apparent range of 2,500 $\mathrm{m}$ becomes easier to be discerned.

We judged the subsurface echo by its linear appearance in B-scan display. On the B-scan display of Fig. 5, however, we recognize other trends of echoes which show straight linear patterns. They look so similar to a subsurface echo that they can be regarded as subsurface echoes as well. Confusion of those surface off-nadir echoes with subsurface echo should be avoided by cross checks with other observations of nearby orbits.

In a year period of operation, SELENE will make about 


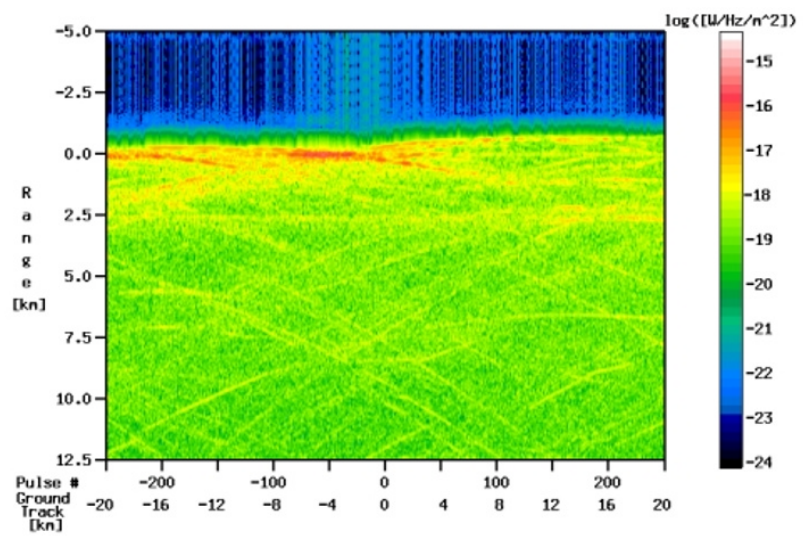

Fig. 4. A B-scan display of the case of slightly lossy subsurface material. The range data is offset $100 \mathrm{~km}$ so that the zero range coincides with the nadir point on the mean surface. Subsurface echo is easily recognized as a straight linear pattern at the apparent depth of $2,500 \mathrm{~m}(2.5 \mathrm{~km})$.

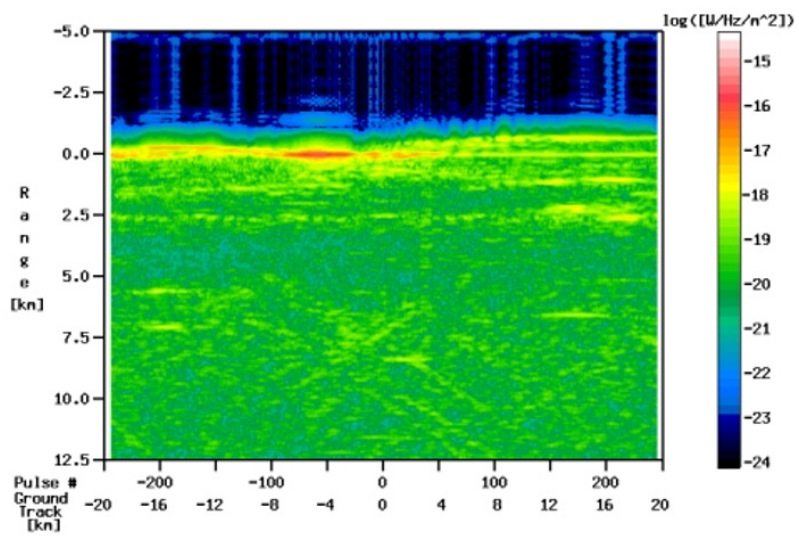

Fig. 5. A B-scan display of 11 data stacking of the case of slightly lossy subsurface material. Note that the intensity of surface off-nadir echoes is reduced.

6000 revolutions around the Moon, which leads to that the mean spatial interval of orbits at equator is $1.6 \mathrm{~km}$. Thus, LRS observations of the same area from adjacent orbits should be available. In those observations, off-nadir echo would appear in different ranges in the B-scan displays of different orbits, depending on the geometry of the echo target positions and the SELENE orbits, while subsurface boundary interface echo would appear in the same range even in different orbits because the spatial gradient of subsurface boundary interface is expected to be very small.

An alternative technique of the cross check is Synthetic Aperture Radar (SAR) technique. SAR analysis could determine the position of a surface echo target. Comparison of a SAR image with an optical image would be a strong help to determine the target location of the echo of concern.

\subsection{LRS observation through moderately lossy subsur- face material}

The dielectric constant of subsurface material in the upper layer for the case of moderately lossy media is assumed as

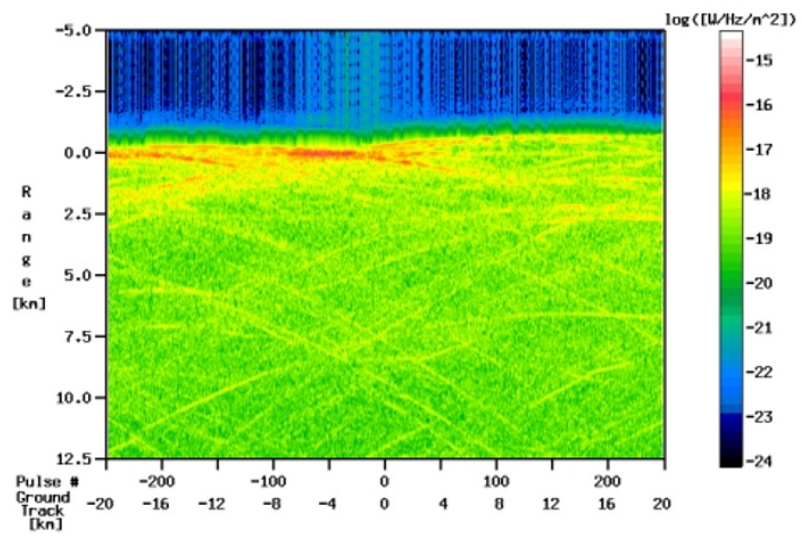

Fig. 6. A B-scan display of the case of moderately lossy subsurface material. The subsurface echo is hardly recognized due to the propagation attenuation in the subsurface material.

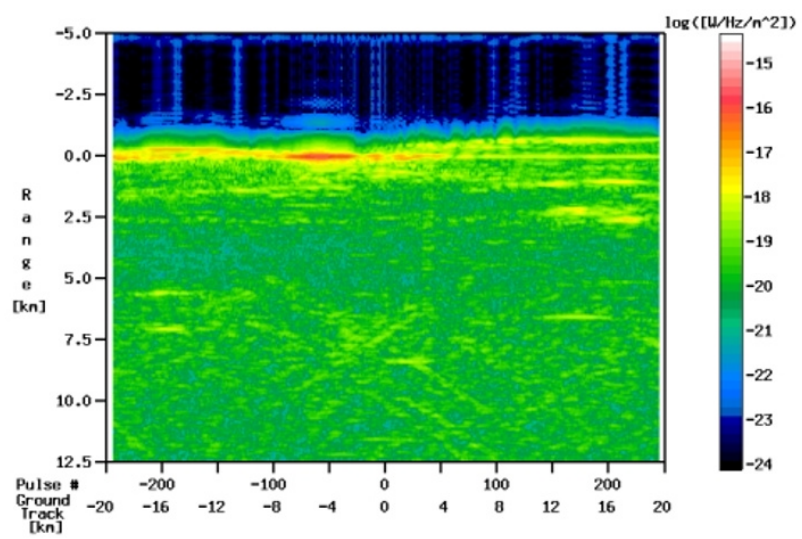

Fig. 7. A B-scan display of 11 data stack of the case of moderately lossy subsurface material. The subsurface echo appears at the apparent depth of $2,500 \mathrm{~m}(2.5 \mathrm{~km})$, which is hardly recognized in the single pulse B-scan display of Fig. 6.

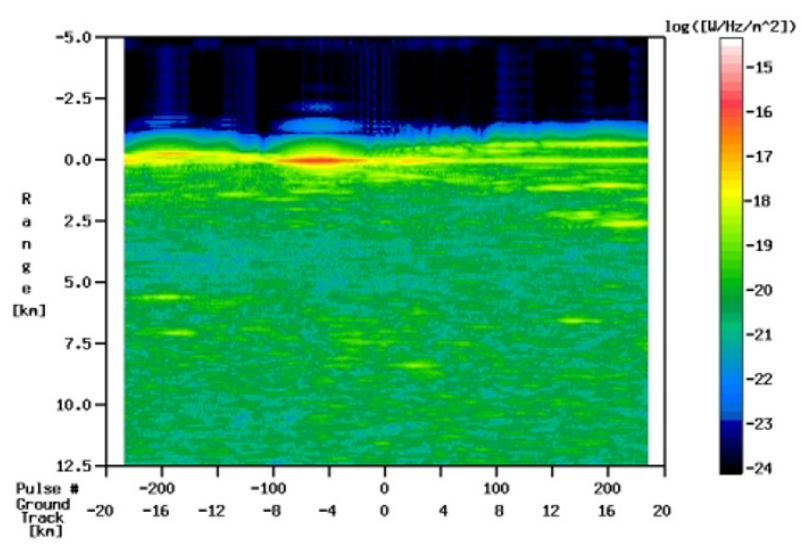

Fig. 8. A B-scan display of 31 data stack of the case of moderately lossy subsurface material. Note that the subsurface echo disappears: excess data stack spoils the subsurface signal. This implies that there is an optimal limit for data stacking.

$$
\varepsilon_{1}=4.0+i 0.03
$$


thus loss $\tan \delta$ is 0.0075 . Other conditions are common to the previous case.

In this case of moderately lossy subsurface material, subsurface echo is hardly seen in the B-scan display as given in Fig. 6, because the subsurface nadir echo is much weaker than that of previous case due to stronger attenuation during subsurface propagation. Therefore, in order to detect the subsurface echo, the surface clutter intensity must be reduced by applying the data stacking technique.

Figure 7 shows the B-scan display of 11 stack data. In the B-scan display, intensity of surface off-nadir echo has been reduced and subsurface echo appears at the apparent range of $2,500 \mathrm{~m}$. The technique certainly works on the detection of the subsurface echo. However, further stacking of data has been found to spoil subsurface reflection signals. Figure 8 shows the B-scan of 31 stack data, in which the subsurface echo cannot be discerned at the apparent range of $2,500 \mathrm{~m}$.

Together with the previous B-scan results of Fig. 7, it is deduced that the data stacking technique has a certain limit of application to detect subsurface echo. Thus, our interest is in how to make out the limit, which is discussed in the next section.

\section{Data Analysis Methodology for LRS Observa- tion in Highland Region}

We have confirmed that data stacking is an effective technique to reduce the surface clutters in order to detect subsurface echo, however, on the other hand, we have seen that excess stacking of data spoils subsurface signal as well. Those facts imply that there is an optimal data stack limit for the technique. We derive the optimal data stack limit by considering a simple model of data stacking technique.

\subsection{Optimal data stack limit}

Let us consider a simple model of LRS observation shown in Fig. 9. In the figure, subsurface boundary interface is a smooth plane and the subsurface space between the surface and subsurface boundary interface is filled with uniform lunar material whose dielectric constant is $\varepsilon_{1}$. Geometrical range to the nadir point of subsurface interface is assumed to be $R_{0}+R_{s u b}$. LRS observation is carried out at two points, point 1 and point 2 . At point 1 , the surface nadir range is $R_{0}$ and the geometrical subsurface range is $R_{s u b}$. At point 2 , the surface range is $R_{0}-\Delta R$ and the geometrical subsurface range is $R_{s u b}+\Delta R$, where $\Delta R$ is the elevation difference of surface terrain from the nadir point of point 1 .

The apparent range of the subsurface echo observed at point 1 is

$$
R_{1}=R_{0}+\sqrt{\Re \varepsilon_{1}} R_{\text {sub }}
$$

whereas the apparent range of the subsurface echo observed at point 2 is

$$
R_{2}=R_{0}-\Delta R+\sqrt{\Re \varepsilon_{1}}\left(R_{s u b}+\Delta R\right) .
$$

Therefore, the difference of those apparent ranges is

$$
R_{2}-R_{1}=\left(\sqrt{\Re \varepsilon_{1}}-1\right) \Delta R
$$

hence, taking into account that the received pulse has made a round trip to the target, the phase difference $\Delta \phi$ of subsur-

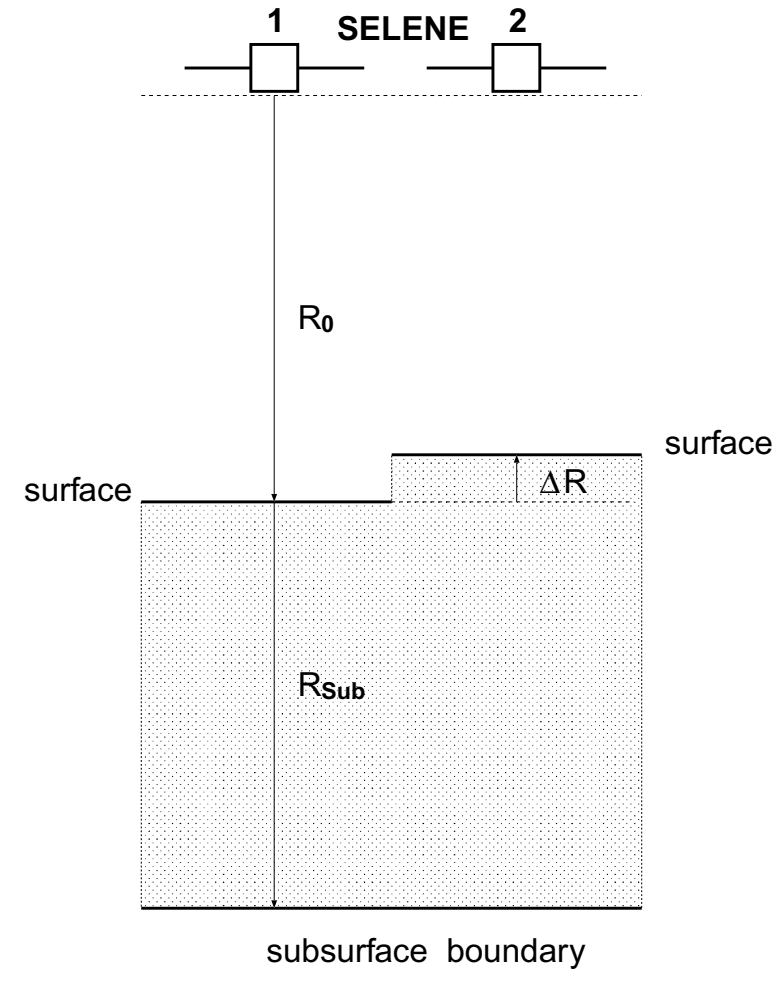

Fig. 9. A simple model of LRS subsurface sounding.

face echoes of those two observations is

$$
\Delta \phi=2 \pi \frac{2\left(\sqrt{\Re \varepsilon_{1}}-1\right) \Delta R}{\lambda}
$$

where $\lambda$ is the wavelength of LRS transmission pulse in vacuum.

In order to make data stacking be carried out in in phase sense, or in constructive sense, the phase difference $|\Delta \phi|$ should satisfy the condition

$$
\left|2 \pi \frac{2\left(\sqrt{\Re \varepsilon_{1}}-1\right) \Delta R}{\lambda}\right|<\frac{1}{2} \pi .
$$

Thus, $\Delta R$ should satisfies the condition

$$
-\frac{\lambda}{8\left(\sqrt{\Re \varepsilon_{1}}-1\right)}<\Delta R<\frac{\lambda}{8\left(\sqrt{\Re \varepsilon_{1}}-1\right)},
$$

therefore, the maximum elevation difference allowed to make in phase sense data stacking for the subsurface nadir echo is

$$
\Delta R_{\max }=\frac{\lambda}{4\left(\sqrt{\Re \varepsilon_{1}}-1\right)} .
$$

We then apply (17) to the present simulation to find the in phase stack limit for those virtual LRS observations. Because the real part of dielectric constant of the subsurface material in the simulation is 4.0 , the optimal data stacking limit (17) is

$$
\Delta R_{\max }=15 \mathrm{~m} .
$$

Therefore, the width of the data stack window should be chosen as such period that the elevation variation of nadir 


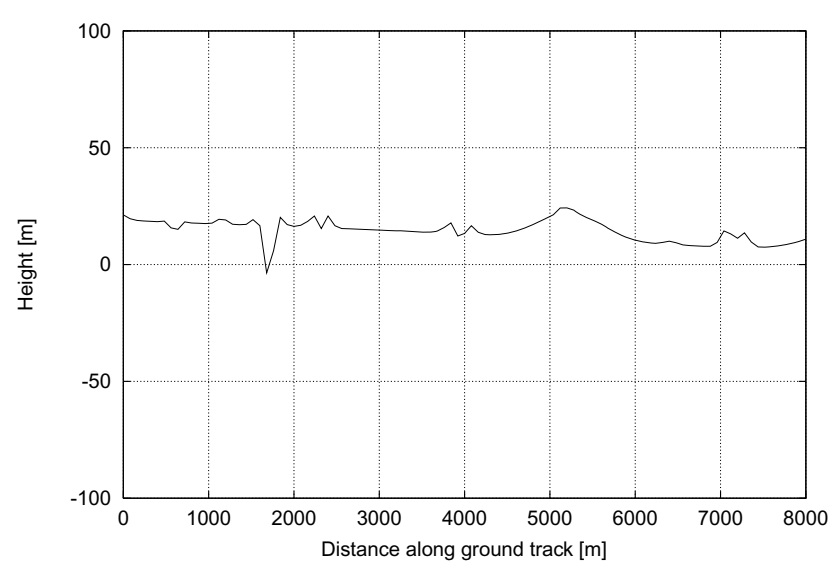

Fig. 10. Height distribution of the surface along the ground track.

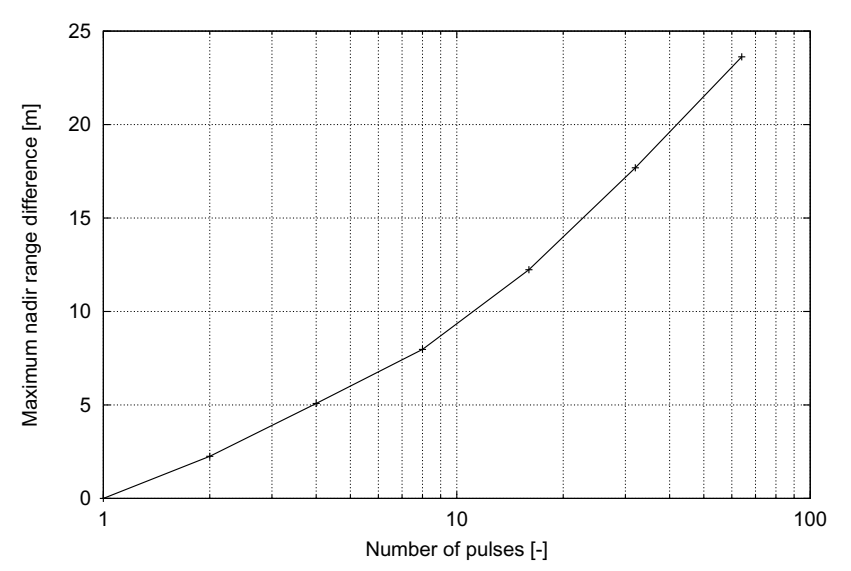

Fig. 11. Mean value of the maximum range difference. Note that in phase stack limit, 15 [m], was reached at the data stack number of about 20.

surface points remains within $15 \mathrm{~m}$, in order to realize in phase sense data stack.

Figure 10 shows the elevation distribution of the surface terrain along the ground track of SELENE spacecraft in the simulation model. During each transmission interval, the spacecraft moves along the orbit for $80 \mathrm{~m}$. The extent of horizontal axis $(8,000 \mathrm{~m})$ of Fig. 10 corresponds to the period of 100 successive sounding operations.

Figure 11 shows a plot of the mean value of the maximum elevation difference of nadir points of the surface in data stacking windows as a function of data stack number. The width of window is represented by the number of data stack. The maximum elevation difference in each data stacking window period was found from the DEM data of the surface model of the simulation. The windows were set in the same manner as those for running average. The plot shows, as is expected, the maximum elevation difference increases as data stack number increases. Around the data stack number of 20, the maximum elevation difference reaches the in phase stack limit, $15 \mathrm{~m}$. Thus, it is expected that data stacking technique is effective until the data stack number reaches 20 for the present case of LRS observation.

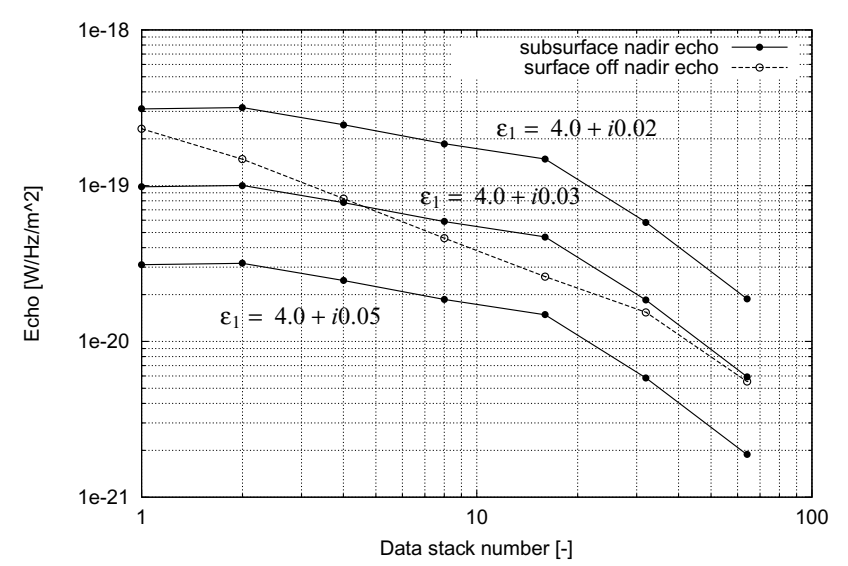

Fig. 12. Effect of data stack on intensities of subsurface nadir echo and surface off-nadir echo.

\subsection{Verification of the optimal data stack limit}

In order to verify the optimal data stack limit obtained above, we investigate the effect of data stack on subsurface echo. Figure 12 shows the variation of subsurface echo intensity as functions of data stack number (solid lines), and the variation of the intensity of surface off-nadir echo (dashed line) of the same range as the apparent range of the subsurface echo. The subsurface echo intensity is plotted for three cases of " $\varepsilon_{1}=4.0+i 0.02$ ", " $\varepsilon_{1}=4.0+i 0.03$ ", and " $\varepsilon_{1}=4.0+i 0.05$ ". The surface echo is of the case of slightly lossy subsurface material, " $\varepsilon_{1}=4.0+i 0.02$ ".

Rapid decrease of surface off-nadir echo intensity in Fig. 12 implies that surface off-nadir echo has been stacked in out of phase sense. On the other hand, the slow change of subsurface echo intensities implies that subsurface echo signals were stacked in in phase sense until stack number reaches 16 , over which data stack turned to be out of phase sense stack thus intensity variations show rapid decrease. These are consistent with our observations on B-scan displays as the results of data stacking process.

For the case of slightly lossy subsurface material, $\varepsilon_{1}=$ $4.0+i 0.02$, the intensity of subsurface echo is greater than the surface off-nadir echo even before data stacking, therefore, the subsurface echo is detected in the B-scan display of single pulse A-scope data (Fig. 4). And as the plot shows, 11 data stacking makes their intensity difference greater, which leads to easier recognition of the subsurface echo in B-scan display as shown in Fig. 5.

For the case of moderately lossy subsurface material, $\varepsilon_{1}=$ $4.0+i 0.03$, the mean intensity of subsurface echo is smaller than the mean intensity of surface off-nadir echo therefore the subsurface echo is hardly detected on the B-scan display before data stacking (Fig. 6). However, as Fig. 12 shows, the intensity of subsurface echo turns to be greater than the surface off-nadir echo after 11 data stacking, thus the subsurface echo is detected in the B-scan display of Fig. 7. But, excess data stack such as 31 data stack spoils the subsurface echo and it again disappears in the B-scan display of Fig. 8.

For the case of seriously lossy subsurface material, $\varepsilon_{1}=$ $4.0+i 0.05$, (of which B-scan display is not presented in this paper) the intensity of the subsurface echo is never greater than the surface off-nadir echo at any number of data stack, 
thus, it is impossible to detect the subsurface echo.

\subsection{Determination of the optimal data stack limit}

Now that our interest is in how to find the optimal data stack limit in practice. Because it is impossible to determine the optimal number of data stack in prior to the observation, for we have neither knowledge of detail elevation distribution of the surface nor of dielectric constant of subsurface material in the region of concern, a method should be established, which enables us to avoid wasting time on looking for subsurface echoes that are impossible to be detected.

A method to estimate the optimal data stack limit is found by giving a consideration to the effect of data stacking on both the surface nadir echo and the subsurface echo.

First we consider the in phase stack condition for surface nadir echo. Using the same simple model as we have used in considering the optimal condition of data stacking for subsurface echo detection, we can derive the in phase stack limit for surface nadir echo as

$$
\Delta R_{0, \max }=\frac{\lambda}{4}
$$

therefore

$$
\Delta R_{0, \max }=15 \mathrm{~m}
$$

for the present simulation. Validity of this condition is verified by analysing the behavior of surface nadir echo intensity in a similar fashion to the analysis of subsurface nadir echo intensity of Fig. 12. The result is presented in Fig. 13 where the intensity of the surface nadir echo shows a very slow decrease as a function of data stack number until the stack number reaches 16 , over which the decrease turns to be rapid. The behavior of the surface nadir echo intensity is essentially the same as those of subsurface off-nadir echo.

In the present simulation, the real part of the dielectric constant of subsurface media, $\Re \varepsilon_{1}$ is assumed to be 4.0 . Thus the in phase sense stack limit for the subsurface echo, (17), coincides with that of surface nadir echo, (19). In fact, the intensities of surface nadir echo and subsurface nadir echoes show the almost same behavior as functions of data stack number. This means that, from the behavior of surface nadir echo intensity as a function of data stack number, we can estimate the number of optimal data stack limit as the number of stack over which the intensity shows a rapid decrease.

In a rigorous sense, this estimation is given to the case of the condition

$$
\Re \varepsilon_{1}=4.0 .
$$

From the measurement of returned samples, however, lunar solid rock has been found to have dielectric constant whose real part tends to be larger than 4.0 (Olhoeft and Strangway, 1975). This leads to that the optimal data stack limit of (18), thus (20), would be less precise condition in most cases of LRS observations in practice. Therefore, allowing some uncertainty that derives from the lack of information about the dielectric constant of subsurface media, we may adopt (20) as the optimal data stack limit.

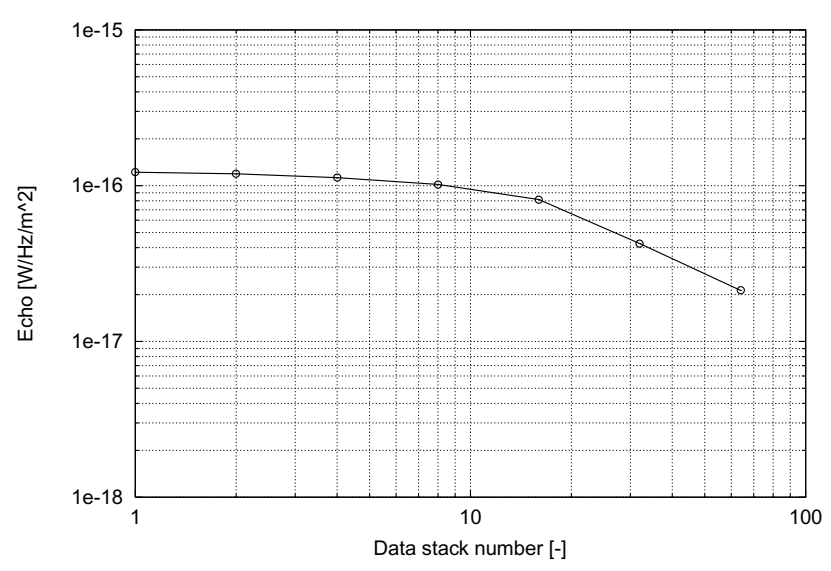

Fig. 13. Effect of data stack on the intensity of surface nadir echo.

\subsection{Data analysis methodology for LRS observation in a highland region}

From considerations given above, we establish a data analysis methodology for LRS observation in highland region as follows:

(1) Make B-scan display of LRS observation.

(2) Try data stacking technique if subsurface echo is not detected after (1).

(3) Increase the number of data stack until the optimal data stack limit.

(4) The optimal data stack limit is found as such that surface nadir echo intensity starts rapid decrease as a function of data stack number.

(5) If subsurface echo is not yet detected even when the optimal data stack limit has been reached, the subsurface echo is not detectable.

\section{Conclusive Remarks}

A data analysis technique for subsurface sounding observation of LRS in a highland region has been studied in the purpose of establishing a data analysis methodology so as to maximize the detectability of signal from subsurface boundary interface. Using a numerically generated surface feature of highland region which is characterized by impact craters, whole sequence of LRS observations of a highland region and data analyses have been simulated by the KiSS code.

The established data analysis methodology consists of a set of data analysis techniques, and gives a guide line to determine the detectability of subsurface signal. The points of the methodology are (1) the subsurface echo signal is recognized as a straight linear pattern in a B-scan display, (2) data stacking technique is introduced to reduce surface off-nadir echoes, however, (3) the data stacking should be done within the range of optimal data stacking, and, (4) the limit of optimal data stacking can be found from the behavior of the surface nadir echo.

The established methodology will be the standard data analysis methodology of LRS and will enable efficient and effective analysis of large amount of LRS observation data.

Spaceborne planetary sounding is recently recognized as a powerful tool of planetary science. Japanese Martian exploration project, Nozomi, is on its way to Mars carrying an HF sounder that carries out altimetry observation of Martian 
surface in HF band (9 MHz) (Oya and Ono, 1998) as well as plasma sounder experiment. Mars Express project of EU will also carry an HF sounder in order to investigate Martian surface/subsurface along with its ionospheric sounding $(\mathrm{Pi}-$ cardi et al., 2000). And, Europa orbiter project of US will be equipped with sounding radar so that it investigate the subsurface of Europa, an icy satellite of Jupiter, in search of possible subsurface ocean (Maddock et al., 1999). In either mission, the data analysis methodology of LRS may be applied with some modification that is required to meet the specific condition of the objective. For example, sounding observation of Martian surface/subsurface would not properly be done without considering the dispersion effect of Martian ionosphere on the transmission sounder pulse. The KiSS code would be utilized to find solutions to meet those specific conditions, and, once observation data are obtained, to help interpreting them as well.

Acknowledgments. The present research was carried out as a basic development study of the Lunar Radar Sounder experiment by SELENE project. The KiSS code has been developed as a collaboration with Super Computing System, Information Synergy Center, Tohoku University. The authors would like to thank them for their consultation and advice on development of the KiSS code. And the authors appreciate Dr. T. Okada and the anonymous referee for their careful reading of the manuscript and their advice.

\section{References}

Baldwin, R. B., Lunar crater count, Astronomical Journal, 69, 377, 1964. Kobayashi, T., H. Oya, and T. Ono, A-scope analysis of subsurface radar sounding of lunar mare region, Earth Planets Space, 54, this issue, 973982, 2002.

Maddock, R. W., K. B. Clark, C. A. Henry, and P. J. Hoffman, The outer planets/solar probe project: Between an ocean, a rock, and a hot place, in 1999 IEEE Aerospace Conference. Proceedings vol. 1, pp. 383-402, IEEE, Piscataway, NJ, USA, 1999.

McGill, G. E., Morphology of lunar craters: a test of lunar erosional models, ICARUS, 21, 437-447, 1974.

Olhoeft, G. R. and D. W. Strangway, Dielectric properties of the first 100 meters of the moon, Earth Planet. Sci. Lett., 24, 394-404, 1975.

Oya, H. and T. Ono, A new altimeter for Mars land shape observations utilizing the ionospheric sounder system onboard the Planet-B spacecraft, Earth Planets Space, 50, 229-234, 1998.

Phillips, R. J., G. F. Adams, Jr., W. E. Brown, R. E. Eggleton, P. Jackson, R. Jordan, W. I. Linlor, W. J. Peeples, L. J. Porcello, J. Ryu, G. Schaber, W. R. Sill, T. W. Thompson, S. H. Ward, and J. S. Zelenka, Apollo Lunar Sounder Experiment, Chap. 22, Apollo 17 Preliminary Science Report, NASA SP-330, 1973.

Picardi, G., S. Sorge, R. Seu, R. Orosei, C. Zelli, and E. Zampolini, The subsurface investigation by Mars Advanced Radar for subsurface and ionosphere sounding (MARSIS), in IGARSS 2000. Proceedings vol. 4, pp. 1730-1732, IEEE, Piscataway, NJ, USA, 2000.

Pike, R. J., Depth/diameter relations of fresh lunar craters: revision from spacecraft data, Geophys. Res. Lett., 1, 291, 1974.

T. Kobayashi (e-mail: tak@stpp1.geophys.tohoku.ac.jp), H. Oya, and T. 